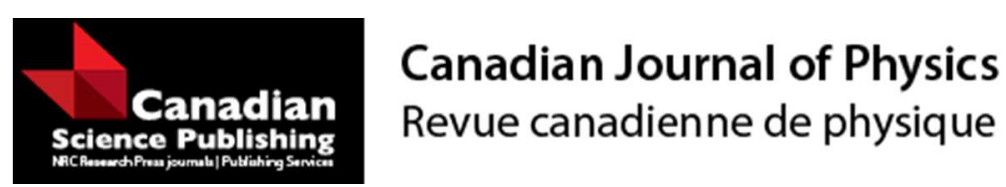

\title{
Regularized perturbative series for the ionization potential of atomic ions
}

\begin{tabular}{|r|l|}
\hline Journal: & Canadian Journal of Physics \\
\hline Manuscript ID & cjp-2016-0255.R1 \\
\hline Danuscript Type: & Article \\
\hline Complete List of Authors: & $\begin{array}{l}\text { Gil, Gabriel; Instituto de Cibernetica Matematica y Fisica; Istituto } \\
\text { Nanoscienze Consiglio Nazionale delle Ricerche; Universita degli Studi di } \\
\text { Modena e Reggio Emilia, Dipartimento di Scienze Fisiche, Informatiche e } \\
\text { Matematiche } \\
\text { González, Augusto; Instituto de Cibernetica Matematica y Fisica }\end{array}$ \\
\hline Keyword: & $\begin{array}{l}\text { atomic ions, ionization potential, regularized perturbation theory, large-Z } \\
\text { expansion, anionic instability threshold }\end{array}$ \\
\hline & \\
\hline
\end{tabular}

SCHOLARONE

Manuscripts 


\title{
Regularized perturbative series for the ionization potential of atomic ions
}

\author{
Gabriel Gil ${ }^{1,2,3}$ and Augusto Gonzalez ${ }^{1}$ \\ ${ }^{1}$ Instituto de Cibernética, Matemática y Física, Havana, Cuba, \\ ${ }^{2}$ S3, CNR-Istituto di Nanoscienze, Modena, Italy, and \\ ${ }^{3}$ Dipartimento di Scienze Fisiche, Informatiche e Matematiche, \\ Universitá degli studi di Modena e Reggio Emilia, Modena, Italy.
}

\begin{abstract}
We study $N$-electron atoms with nuclear charge $Z$. It is well known that, in the cationic $(Z>N)$ large- $Z$ region, the atom behaves as a weakly interacting system. The anionic $(Z<N)$ regime, on the other hand, is characterized by an instability threshold at $Z_{c} \lesssim N-1$, below which the atom spontaneously emits an electron. We construct a regularized perturbative series (RPS) for the ionization potential that is based on the behaviors for large $Z$ and $Z$ near $Z_{c}$. The large- $Z$ expansion coefficients are analytically computed from perturbation theory, whereas the slope of the energy curve at $Z=N-1$ is computed from a kind of zero-range forces theory that uses as input the electron affinity and the covalent radius of the neutral atom with $N-1$ electrons. Relativistic effects in the one-particle Hamiltonian are considered at the level of first-order perturbation theory. Our RPS formula is to be used in order to check the consistency of the ionization potential values for atomic ions contained in the NIST database.
\end{abstract}

PACS numbers: 32.30.-r, 32.10.Hq, 31.15.-p

Keywords: Atomic ions; ionization potential; regularized perturbation theory; large- $Z$ expansion; anionic instability threshold

\section{INTRODUCTION}

Since the foundation of Quantum Mechanics, a huge amount of data on energy levels, linewidths and other atomic properties has been recorded. Very often, such compilations are still waiting for a qualitative analysis, based on simple models.

In recent articles, ${ }^{1,2}$ on the basis of the scaling suggested by Thomas-Fermi theory, ${ }^{3}$ we have demonstrated universality in the ionization potentials and the correlation energies of atomic cations.

In the present paper, we construct an analytical expression for the ionization potential of $N$-electron atoms with arbitrary nuclear charge $Z>N-1$, which is to be used in order to detect problematic values ${ }^{4,5}$ in the numbers provided by the NIST database. ${ }^{6}$

Our expression is a regularized perturbative series (RPS), previously employed in other contexts. ${ }^{7}$ We use perturbation theory in $1 / Z,{ }^{8}$ in order to compute the first two coefficients of the energy series in the large- $Z$ region. ${ }^{9}$ Additionaly, we require our RPS to reproduce the value of the ionization potential at $Z=N-1$ (i.e., the electron affinity) and the slope of the curve at this point. The latter is computed from a kind of zero-range forces theory that uses as input the electron affinity and the covalent radius of the neutral atom with $N-1$ electrons. ${ }^{10}$ The RPS continuously interpolates between the $Z \approx N-1$ and large- $Z$ limits for a given isoelectronic sequence.

Hereunder, we provide the main formulae entering the RPS expression. A few isoelectronic sequences are studied in quality of examples, whereas a systematic analysis of the NIST database is contained in Refs. [4] and [5].

\section{ATOMS NEAR THE ANIONIC INSTABILITY THRESHOLD}

It is well known that, for large $Z$, the attraction of the electrons by the nucleus is stronger than electron-electron repulsion. On the contrary, for the neutral atom both contributions are more or less balanced, and in the anionic domain this balance may even be broken at a given $Z_{c} \lesssim N-1$, where the atom spontaneously autoionizes.

First-principle calculations ${ }^{11}$ and some extrapolations $^{12}$ indicate that $Z_{c}$ is indeed very close to $N-1$, excluding the possibility of doubly charged negative ions. A recent result by Gridnev, ${ }^{13}$ on the other hand, rigorously states that the wave function is normalized at threshold assuming $N-2<Z_{c}<N-1$. If we combine this result with perturbation theory, we get that the binding energy exhibits a linear dependence on $Z$ near $Z_{c}$, under the same hypothesis.

In a previous paper, ${ }^{10}$ we compute the slope of the curve not at $Z_{c}$, but at $Z=N-1$. At this value of $Z$ the outermost electron weakly interacts with the neutral core and the interaction is short-ranged. It can be shown that conditions are fulfilled for the application of zero-range forces theory. ${ }^{14}$ The slope of the curve may be computed from $^{10}$

$$
s=2 \kappa e^{2 \kappa R} \int_{R}^{\infty} d r e^{-2 \kappa r} / r,
$$

where $\kappa=\sqrt{2 E_{a}}$ and $E_{a}$ is the electron affinity of the neutral system with $N-1$ electrons. Atomic units are to be used everywhere in the paper. $R$ is related to the size of the core, containing nuclear charge $Z$ and $N-1$ electrons. For computational purposes, we use the covalent radius of the $N-1$ electron atom as an estimation of $R . E_{a}$ and $R$ are experimental values. ${ }^{15}$ 
Equation (1) will be used in Sec. IV, where we construct a RPS for the ionization potential. Notice that Eq. (1) is not valid for the isoelectronic sequences with an unstable anion, where $E_{a}=0$ and $Z_{c}=N-1$. The interpolation model formulated in Sec. IV inherits this limitation.

Our theory might be generalized for the case of unstable anions if an approximated functional form for the ionization potential, such as the one stemming from quantum defect theory, ${ }^{16}$ is considered.

\section{THE LARGE- $Z$ LIMIT}

In the following, we shall construct the large- $Z$ series (i.e., with $N / Z \rightarrow 0$ ) for the atomic energy. This is, in fact, an unphysical limit. In nature, atomic ions become unstable for large $Z$, showing a threshold for electronpositron pair production at $Z \sim 137 .{ }^{17}$ Performing the electronic coordinate scaling $r_{i} \rightarrow r_{i} / Z$ in the nonrelativistic Hamiltonian, we get

$$
\hat{H}=Z^{2}\left\{-\sum_{i=1}^{N} \frac{\nabla_{i}^{2}}{2}-\sum_{i=1}^{N} \frac{1}{r_{i}}+\frac{1}{Z} \sum_{i<k} \frac{1}{\left|\vec{r}_{i}-\vec{r}_{k}\right|}\right\} .
$$

Notice that the expression inside brackets has a oneparticle contribution (kinetic energy plus nuclear attraction) and the two-particle repulsion between electrons. The latter is of order $1 / Z$. At large values of $Z$, the atom can be described as a system of non-interacting electrons in the central Coulomb field of the nucleus. The energy in this leading approximation is

$$
E^{(0)}=-\sum_{i=1}^{N} \frac{Z^{2}}{2 n_{i}^{2}},
$$

where $n_{i}$ is the principal quantum number corresponding to the $i$-th occupied orbital.

Next, we shall include electron repulsions in first order perturbation theory. The energy is written as

$$
E=E^{(0)}+Z\left\langle\Psi^{(0)}\left|\sum_{i<k} \frac{1}{\left|\vec{r}_{i}-\vec{r}_{k}\right|}\right| \Psi^{(0)}\right\rangle,
$$

where $\Psi^{(0)}$ is a Slater determinant made of Hydrogen electronic wavefunctions. Corrections are explicitly given by

$$
\begin{aligned}
E^{(1)} & =Z\left\langle\Psi^{(0)}\left|\sum_{i<k} \frac{1}{\left|\vec{r}_{i}-\vec{r}_{k}\right|}\right| \Psi^{(0)}\right\rangle \\
& =Z \sum_{i<k}^{N}\{\langle i k \| i k\rangle-\langle i k|| k i\rangle\} .
\end{aligned}
$$

Note that the sums run over the occupied states $|i\rangle$ and $|k\rangle$ in the Slater determinant, and that $\langle i k \| i k\rangle$ and $\langle i k \| k i\rangle$ denote, respectively, direct and exchange twoelectron Coulomb integrals involving orbitals $i$ and $k$. Their explicit expression can be found in Ref. [18].

Once we constructed a series for the ground state total energy (with $N$ electrons occupying the lowest-energy orbitals),

$$
E_{g s}=a_{2} Z^{2}+a_{1} Z+\ldots
$$

we can find a similar expression for the ionization potential, defined as $I_{p}(N, Z)=E_{g s}(N-1, Z)-E_{g s}(N, Z)$. We get $^{19}$

$$
I_{p}=b_{2} Z^{2}+b_{1} Z+\ldots
$$

where,

$$
\begin{aligned}
& b_{2}=\frac{1}{2 n_{N}^{2}}, \\
& b_{1}=-\sum_{i=1}^{N}\{\langle N i \| N i\rangle-\langle N i \| i N\rangle\} .
\end{aligned}
$$

In these equations, $n_{N}$ is the principal quantum number of the last electronic shell, and $|N\rangle$ is the last occupied orbital.

We shall stress that, in the non-interacting regime, the ground state of a full-shell atom is exactly nondegenerate. On the other hand, the degeneracy of the ground state of an open-shell system gets resolved in the weakly-interacting regime. Hund's rules determine the occupation of the states in last shell so as to minimize the interaction energy. Applying these rules, we obtain a uniquely defined $\Psi_{g s}^{(0)}$, i.e., a Slater determinant composed by the $N$ lowest-energy orbitals. The notion of the last occupied orbital is unambiguous due to the Hund rules filling. ${ }^{20}$

The scheme obtained by partnering non-degenerate perturbation theory and Hund's rules is approximated. In principle, we should use a degenerate perturbation theory, where the coefficient $a_{1}$ in Eq. (6) comes from the lowest eigenvalue of the two-particle Coulomb repulsion operator in the basis of the Slater determinants belonging to the degeneracy subspace. ${ }^{21}$ The results stemming from non-degenerate perturbation theory partnered with Hund's rules (Eq. (4)) departs from the degenerate perturbation theory ones, in the sense that the former does not reproduce the correct $Z$-dependence of the next-toleading correction to the ground state energy. However, we choose the approximated scheme because of its simplicity and to the immediate inclusion of single-particle relativistic corrections within it (Sec. III A).

To end up this section, we will examine the large- $N$ dependence for $b_{2}$ and $b_{1}$, keeping $Z \gg N$. Let us start 
from the expression for the number of particles of a fullshell system in the non-interacting regime:

$$
N=\sum_{n=1}^{n_{N}} 2 n^{2}=\frac{1}{3} n_{N}\left(n_{N}+1\right)\left(2 n_{N}+1\right) \approx \frac{2}{3} n_{N}^{3},
$$

where we used the degeneracy of the $n$-th shell, i.e., $g_{n}=$ $2 n^{2}$. After substituting $n_{N} \approx(3 / 2)^{1 / 3} N^{1 / 3}$ in Eq. (7a) we get the leading term of the large- $N$ series,

$$
b_{2}=\frac{1}{2}\left(\frac{2}{3}\right)^{2 / 3} N^{-2 / 3}+\ldots
$$

To get the dependence of $b_{1}$ we fitted the functional form $b N^{\beta}$ to the results coming from Eq. (7b) for full-shell systems with $28 \leq N \leq 408$; yielding

$$
b_{1}=-0.72 N^{1 / 3}+\ldots
$$

Equations (9) are consistent with the dependence $I_{p} \approx$ $Z^{2} N^{-2 / 3} f(N / Z)$, suggested by Thomas-Fermi theory. ${ }^{1}$

\section{A. Relativistic corrections}

At large $Z$, a relativistic approach is required. In the leading approximation, one should solve the Dirac equation for an electron in a central Coulomb field. We choose a simpler approach in which relativistic corrections are computed in first order degenerate perturbation theory:

$$
\begin{aligned}
\bar{\varepsilon}_{\mathrm{I}} & =\varepsilon_{\mathrm{I}}+\left\langle\mathrm{I}\left|\hat{V}_{\text {rel }}\right| \mathrm{I}\right\rangle \\
|\overline{\mathrm{I}}\rangle & =|\mathrm{I}\rangle+\sum_{\mathrm{K} \in S_{\perp}^{(\mathrm{I})}} \frac{|\mathrm{K}\rangle\left\langle\mathrm{K}\left|\hat{V}_{r e l}\right| \mathrm{I}\right\rangle}{\varepsilon_{\mathrm{I}}-\varepsilon_{\mathrm{K}}},
\end{aligned}
$$

where $\bar{\varepsilon}_{\mathrm{I}}$ and $|\overline{\mathrm{I}}\rangle$ are the relativistically corrected singleparticle energies and states, respectively, while $\varepsilon_{\mathrm{I}}$ and $|\mathrm{I}\rangle$ are the zero-order (nonrelativistic) equivalents. $|\mathrm{I}\rangle$ is a state for which the total angular momentum (orbital plus spin) is a good quantum number. The relativistic perturbation, $\hat{V}_{\text {rel }}$, includes the kinetic energy correction, the spin-orbit, and the Darwin terms (see Appendix). ${ }^{22}$ The sum in Eq. (10b) runs over the space orthogonal to $|\mathrm{I}\rangle$.

The $b_{2}$ coefficient must be changed in accordance with Eq. (10a). Since $b_{2}=\bar{\varepsilon}_{\mathrm{N}} / Z^{2}$, the final expression reads: ${ }^{22}$

$$
b_{2}=\frac{1}{2 n_{N}^{2}}\left[1+\frac{(Z \alpha)^{2}}{n_{N}^{2}}\left(\frac{n_{N}}{j_{N}+1 / 2}-\frac{3}{4}\right)\right],
$$

where $j_{N}$ is the total angular momentum quantum number of the last occupied state and $\alpha \approx 1 / 137$ is the fine structure constant.

The first order correction to $b_{1}$ can be obtained by replacing $|i\rangle$ and $|N\rangle$ in Eq. (7b) by the expression (10b) and scaling by $Z$. We re-write the final expression in terms of the nonrelativistic states $|i\rangle$ and energies $\epsilon_{i}$ corresponding to Hydrogen; yielding

$$
\begin{aligned}
b_{1}= & -\sum_{i=1}^{N}\{\langle N i \| N i\rangle-\langle N i \| i N\rangle\}+ \\
& -2(Z \alpha)^{2} \sum_{i=1}^{N-1}\left\{\sum_{k \in S_{\perp}^{(N)}} \frac{\langle N i \| k i\rangle-\langle N i \| i k\rangle}{\epsilon_{N}-\epsilon_{k}}\left\langle k\left|\hat{v}_{r e l}\right| N\right\rangle+\sum_{k \in S_{\perp}^{(i)}} \frac{\langle N i \| N k\rangle-\langle N i \| k N\rangle}{\epsilon_{i}-\epsilon_{k}}\left\langle k\left|\hat{v}_{r e l}\right| i\right\rangle\right\},
\end{aligned}
$$

where $\hat{v}_{r e l} \equiv \hat{V}_{r e l}(Z=1, \alpha=1)$. In Eqs. (11), the dependence on $Z$ and $\alpha$ is explicit, and the nonrelativistic limit of Eqs. (7) is recovered once $(Z \alpha)^{2} \rightarrow 0$.

A summary of matrix elements $\left\langle k\left|\hat{v}_{\text {rel }}\right| i\right\rangle$ is given in the Appendix. Details on the derivation of $\left\langle i\left|\hat{v}_{\text {rel }}\right| i\right\rangle$ can be found in Ref. [22].

Notice that all relativistic corrections considered here are related to one-particle effects. The two-particle corrections, e.g., Darwin, spin-spin contact, orbit-orbit, spin-other-orbit, and spin-spin interactions, entering in the Breit-Pauli Hamiltonian, ${ }^{23}$ may be of the same order of Coulomb repulsion for large- $Z$, but they are not expected to contribute to the ionization potential in the large- $N$ regime (keeping $N / Z \rightarrow 0$ ) because of their scaling with $N{ }^{24}$ For small $N$, this is not the case and indeed the inclusion of two-particle relativistic effects in a perturbation scheme is still required.$^{25}$ Nevertheless, the formulation of our RPS with only one-particle relativistic effects is quite enough to reproduce qualitative features in the large- $Z$ region. Furthermore, higher-order perturbation theory is not within the scope of this article. 


\section{REGULARIZING THE PERTURBATIVE SERIES}

Once the region near the anionic threshold and the large- $Z$ limit are described, one may try to find an interpolation between them. To this end, we use a regularization of the perturbative series, Eq. (7). ${ }^{7}$ The next two formal terms of the series

$$
I_{p}=b_{2} Z^{2}+b_{1} Z+b_{0}+\frac{b_{-1}}{Z}
$$

coming, in principle, from higher order perturbative corrections, are instead used to force that, at $Z=N-1$, $I_{p}(N)=E_{a}(N-1)$ and $d I_{p} / d Z=s$ (Eq. (1)). That is,

$$
\begin{gathered}
E_{a}=b_{2}(N-1)^{2}+b_{1}(N-1)+b_{0}+\frac{b_{-1}}{(N-1)}, \\
s=2 b_{2}(N-1)+b_{1}-\frac{b_{-1}}{(N-1)^{2}}
\end{gathered}
$$

Note that, to get the second expression in (13), we have neglected the relativistic corrections to $b_{2}$ and $b_{1}$ as they should be small in the anionic regime. We get a linear system of two equations and two variables $\left(b_{0}\right.$ and $\left.b_{-1}\right)$, yielding

$$
\begin{aligned}
b_{0} & =E_{a}-3 b_{2}(N-1)^{2}-\left(2 b_{1}-s\right)(N-1), \\
b_{-1} & =2 b_{2}(N-1)^{3}+\left(b_{1}-s\right)(N-1)^{2} .
\end{aligned}
$$

\section{DETECTING PROBLEMATIC POINTS IN THE NIST DATABASE}

We would like to show how Eq. (12), with the coefficients $b_{1}$ and $b_{2}$ given in (11b) and (11a), respectively, and $b_{0}$ and $b_{1}$ coming from (14), can be used to detect inconsistencies in the NIST data for the ionization potential of atomic ions. We study four isoelectronic sequences (Figs. 1-3) in quality of examples. An exhaustive revision will be published elsewhere. ${ }^{4,5}$

In Figs. 1-3, we plot the nonrelativistic and the relativistic RPS, along with the NIST data, for these sequences. The lower panels show the difference between the NIST data and our relativistic RPS (NIST-RPS). The mean relative difference is near $0.4 \%$ for $N=10,4 \%$ for $N=28,3 \%$ for $N=29$, and $9 \%$ for $N=60$. In general, the relative differences decay in the large- $Z$ region as it is expected from an interpolant meeting this limit. ${ }^{7}$

We claim that, in spite of the fact that our relativistic RPS does not have spectroscopic precision, abrupt changes in the difference NIST-RPS may be a sign of
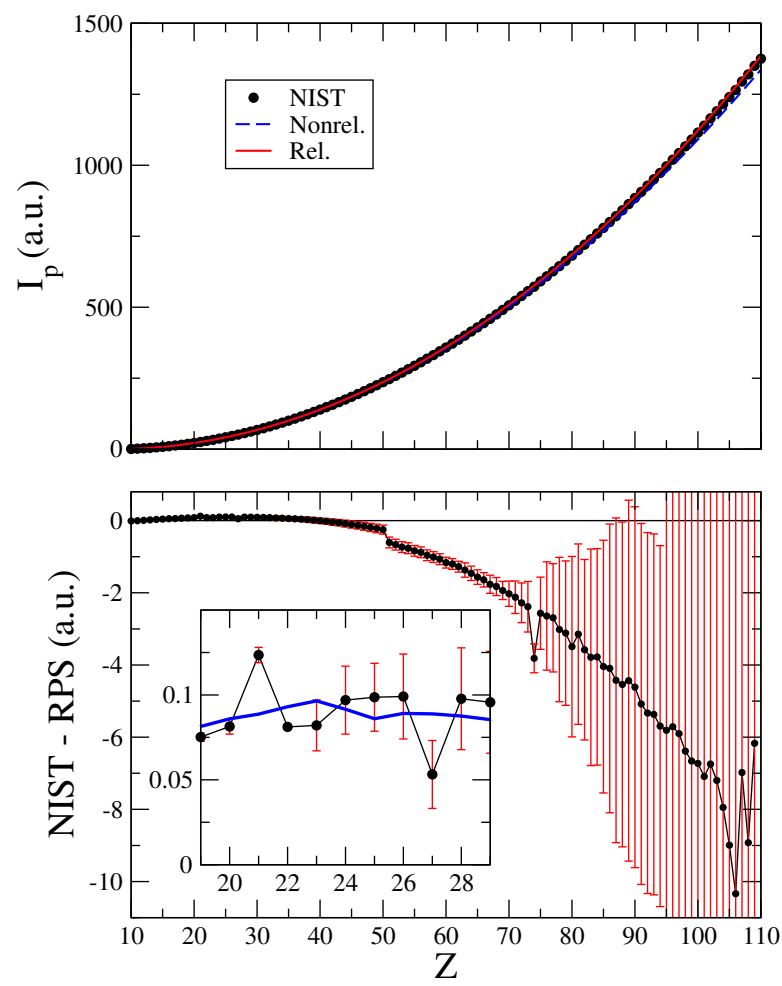

FIG. 1. (Color online) The case of Ne-like ions $(N=10)$. Upper panel: Ionization potential taken from the NIST compilation along with our nonrelativistic (discontinuous, blue) and relativistically corrected (continuous, red) RPS predictions versus atomic charge. Curves seem smooth at any scale. Lower panel: The difference between the NIST reported values and the RPS relativistic curve versus atomic charge. A 5-points running average curve (continuous, blue) is used as a reference for measuring deviations (see the inset). Inconsistencies are detected at $Z=21,22,27,74$ and $Z=50-51$. We stress also that there is a great dispersion of the data for $Z>100$, which is however within the huge uncertainty bars reported for these points.

inconsistency. Indeed, abrupt changes in $I_{p}$ are related to rearrangements of the electronic spectrum. In the interval between rearrangements or for large enough $Z$, the occupancy of orbitals is fixed, ${ }^{20}$ and $I_{p}$ should be smooth. The difference with our smooth RPS interpolant should also be a smooth function of $Z$.

Ne-like systems are closed shell, and do not exhibit rearrangements at any $Z$. In order to make evident inconsistent points in the NIST data, we construct an average NIST-RPS curve by means of a 5 -points running average. In Fig. 1, the $Z=21$ point is so far from the average curve, for example, that it should be corrected. We can even give an estimate of the needed correction by measuring the distance to the average curve, which in this case is -0.034 a.u.

In Ni-like ions, the $3 \mathrm{~d}^{10}$ electronic configuration is reached already for $Z \geq 29$. Thus, we expect a smooth dependence from this point on. The $Z=74$ point, for example, is deviated from the average curve in 0.141 a.u., 
and its uncertainty bar is only 0.115 a.u. wide. Cu-like ions, on the other hand, show a $3 \mathrm{~d}^{10} 4 \mathrm{~s}$ configuration at any $Z$. Nd-like ions experience rearrangements at various
$Z$ values, but in the neighbourhood of $Z=74$, a problematic point, the difference should be smooth. Thus, we can undoubtedly distinguish this point.
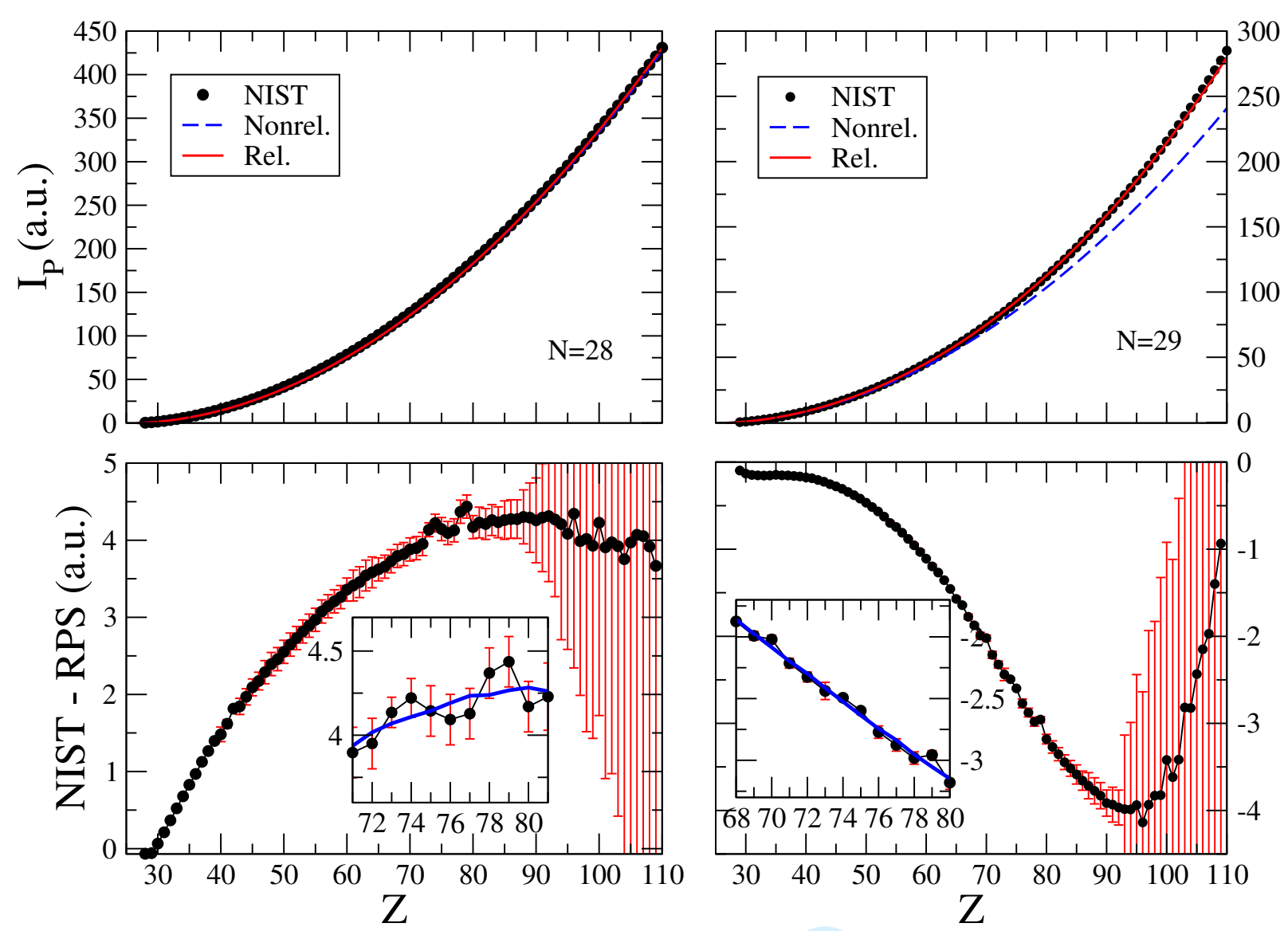

FIG. 2. (Color online) The same as Fig. 1 for $\mathrm{Ni}-(N=28$, left panels $)$ and $\mathrm{Cu}$-like $(N=29$, right panels $)$ ions. In the $N=28$ system, we find inconsistencies at $Z=42,74$, and 79 , whereas for $N=29$ small deviations at $Z=70$, and 79 are noticed. Relativistic effects are significant for $\mathrm{Cu}$-like ions at large $Z$ values, in which the last electron occupies a 4 s orbital.

\section{SUMMARY AND OUTLOOK}

The main result of the present paper is an analytical expression for the ionization potential of $N$-electron atomic ions with nuclear charge $Z \geq N-1$ based on first-principles. This formula is by construction exact at $Z=N-1$, it contains the correct asymptotics for the large- $Z$ region within nonrelativistic quantum mechanical framework, and it continuously interpolates between the two regimes for arbitrary $Z>N-1$. Our methodology stands on two pillars: the $1 / Z$ perturbation expansion, whose leading and next-to-leading orders describe the exact $Z$-dependence of the nonrelativistic groundstate energy for the large- $Z$ domain, and a model for $Z=N-1$ based on the short-range nature of the bound state for the outermost electron and its long-range interaction with an effective core. One-particle relativistic effects are further introduced at the level of first order perturbation theory so as to obtain accurate ionization potential of heavy cations (i.e., for large $Z$ ).

The ionization potential formula, thus derived, enables only to cast the case of atoms with a stable isoelectronic singly-charged anion due to the peculiarities of our zero-range model for the anionic domain $(Z \approx N-1)$. Albeit, the latter is the far most common situation in the periodic table. This drawback can readily be overcomed by considering the diffuse hydrogenic character of 

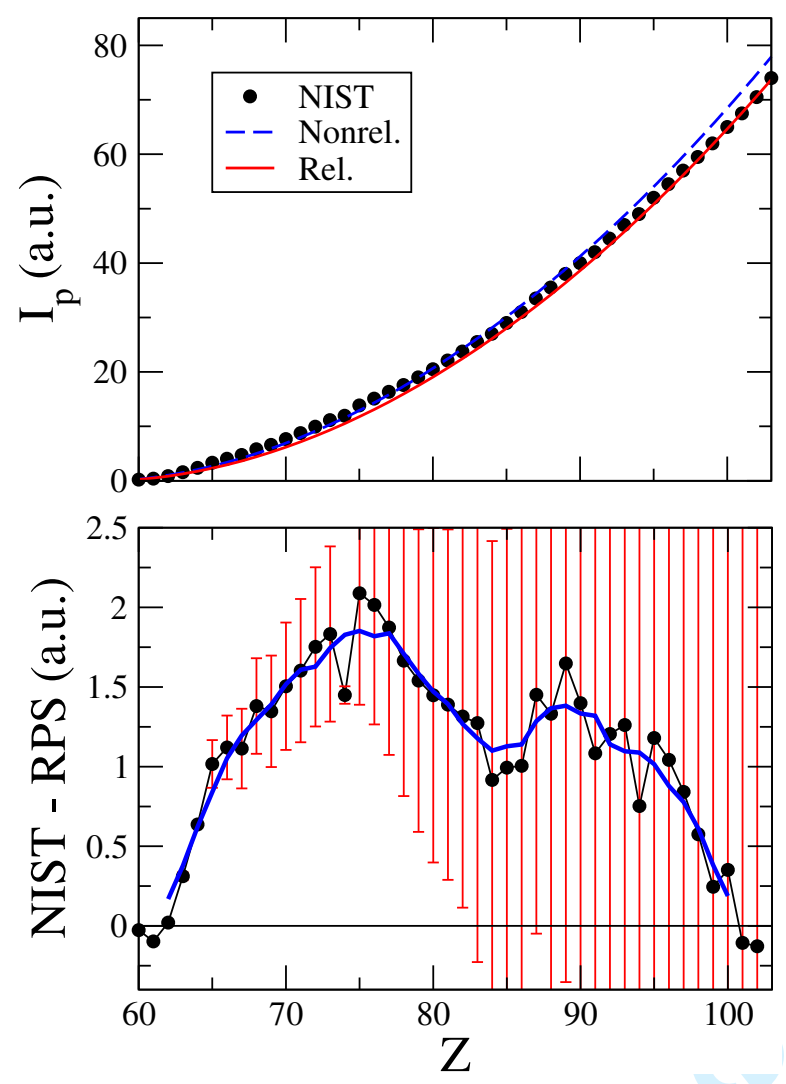

FIG. 3. (Color online) The same as Fig. 1 but for Nd-like ions $(N=60)$. Only the $Z=74$ point is clearly inconsistent.

bound states in systems with unstable isoelectronic anions, through quantum defect ${ }^{16}$ or other sorts of screening theories ${ }^{21}$. Such a development is not within the scope of this article, although it surely motivates further research. Our interpolation scheme (i.e., what we call regularization of the perturbation series in $1 / Z$ ) is well-suited for such amendments if only the number of constraints of the ionization potential in the anionic region (i.e., value, slope, and possibly curvature) equals the number of newly added terms above the next-to-leading order in the $1 / Z$ expansion.

There are two levels of approximation in the calculation of the leading and next-to-leading orders of the $1 / Z$ expansion for the ionization potential. First, the nonrelativistic next-to-leading correction to the ground state energy is computed by non-degenerate perturbation theory, partnered with Hund's rules to unambiguously determine the ground state of open-shell systems. Although Hund's rules are confirmed by NIST sources in the large- $Z$ cationic region, the latter approach is not exact since the coupling between degenerate electronic configurations through Coulomb Hamiltonian may result in a modification of the next-to-leading order coefficient. In principle, degenerate perturbation theory shall supersede the aforementioned approach to account for the right $Z$-dependency near the non-interacting limit $(Z \rightarrow \infty)$. However, we choose the former because it paves the way for a simple introduction of relativistic effects. The second level of approximations regards our relativistic treatment, i.e., based on first-order perturbative corrections to the single-particle energies and states. The perturbation treatment constrains the validity of our asymptotic description for large- $Z$ to a region for which $Z \gg N$ (noninteracting limit within nonrelativistic quantum mechanics) and $(Z \alpha)^{2} \ll 1$ (perturbative relativistic effects). Moreover, two-particle relativistic effects are not considered. Including them within a more consistent generalized perturbation theory in two coupling constants, i.e., $N / Z$ and $(Z \alpha)^{2}$, will be the subject of a new article. ${ }^{25}$

Finally, we compare our ionization potential formula with the NIST data in the case of four isoelectronic series (Ne-, Ni-, Cu- and Nd-like atoms). Although, our regularized perturbation series cannot predict ionization potentials within the chemical accuracy $(\sim 0.11$ a.u. $)$ there is a reasonable agreement with NIST values. The quality of our formula has to be appreciated in two ways: first, the departure from data points (NIST-RPS, in Figs. 13 ) is only a few percent of the total ionization potential in average, and second, it display an overall smooth dependence on $Z$. The former speaks well of the asymptotic descriptions of the two limits that are interpolated. Since our regularized perturbation series is smooth by definition, the latter is a general rule that could only be broken if the data exhibits abrupt changes in the ionization potentials due to rearrangements of the one-particle electronic spectrum. These rearrangements are common near the neutral and anionic regions, as they are closer to the autoionization threshold than to the large- $Z$ noninteracting limit. However, in between rearrangements, non-smooth features in the ionization potential are unexpected. Nevertheless, the difference between NIST data and our regularized perturbation series show rather clear outliers and abrupt jumps in the data that cannot be traced back to rearrangements in the spectrum. Furthermore, very often the expected trend do not even lie within uncertainty bars. Such internal incosistencies are symptoms of erroneous values.

The latter is pointing to a more basic usage of our regularized perturbation series. Rather than predicting new reference data for ionization potentials, that can be accurately calculated or measured with the already existing methods, there is a need of analyzing the enormous amount of compiled data on simple physical grounds. Here is where our model may be helpful, i.e., as a tool for the compilers (e.g., NIST ASD team) to isolate problematic data points that may be associated with actual errors. The internal inconsistencies in the data are apparent once a smooth function, very close to the data points, is taken as a reference. A general scheme of regression, although employing physics informed functional form (e.g., $1 / Z$ expansion with coefficients as fitting parameters), may be insufficient or at least less effective to carry out this task because it relies on all data points, correct and incorrect, a priori indistinguishable. The model of choice shall be as simple as a fit, and enough 
physically meaningful to be confronted with sofisticated $a b$ initio computations or even experiments. We believe our regularized perturbation series is a good candidate to this purpose. A systematic analysis of the ionization potentials data from NIST is presented in Refs. [4] and [5].

\section{ACKNOWLEDGMENTS}

The authors are grateful to the Caribbean Network for Quantum Mechanics, Particles and Fields (ICTP) for support. G.G. also acknowledge financial support from the European Community's FP7 through the Marie Curie ITN-INDEX.

\section{Appendix A: Explicit matrix elements for relativistic corrections}

We can write the relativistically corrected Hamiltonian as $\hat{H}=\hat{H}_{0}+\hat{V}_{\text {rel }}$, where the non-perturbed Hamiltonian is given by $\hat{H}_{0}=-\nabla^{2} / 2-Z / r$, and the perturbation is expressed as a sum of terms:

$$
\begin{aligned}
& \hat{V}_{r e l}=\hat{H}_{1}+\hat{H}_{2}+\hat{H}_{3}, \\
& \hat{H}_{1}=-\frac{\alpha^{2}}{8} \nabla^{4}=-\frac{\alpha^{2}}{2}\left(\hat{H}_{0}+\frac{Z}{r}\right)^{2}, \\
& \hat{H}_{2}=\frac{Z \alpha^{2}}{2} \frac{1}{r^{3}} \hat{\vec{L}} \cdot \hat{\vec{S}}=\frac{Z \alpha^{2}}{4} \frac{1}{r^{3}}\left(\hat{J}^{2}-\hat{L}^{2}-\hat{S}^{2}\right), \\
& \hat{H}_{3}=\pi \frac{Z \alpha^{2}}{2} \delta(\vec{r}),
\end{aligned}
$$

where $\hat{L}^{2}, \hat{S}^{2}$ and $\hat{J}^{2}$ are the orbital, the spin and the total (orbital plus spin) angular momentum operators, respectively.

We consider first-order perturbative corrections due to $\hat{V}_{\text {rel }}(Z, \alpha)$. The relevant matrix element is $\left\langle K\left|\hat{V}_{\text {rel }}\right| I\right\rangle=$ $Z^{4} \alpha^{2}\left\langle k\left|\hat{v}_{r e l}\right| i\right\rangle$, where $\hat{v}_{r e l} \equiv \hat{V}_{r e l}(1,1)$. The involved states $|i\rangle$ are eigenstates of the Hydrogen Hamiltonian ( $\hat{H}_{0}$ with $Z=1$ ), $\hat{L}^{2}, \hat{S}^{2}, \hat{J}^{2}$ and $\hat{J}_{z}$ (z-component of the angular momentum operator $\hat{\vec{J}})$. Only states such that $\langle k \mid i\rangle=0$ enter Eq. (10b), thus we restrict ourselves to this case. We have

$$
\left\langle k\left|\hat{v}_{r e l}\right| i\right\rangle=\frac{1}{2}\left[-\langle k|1 / r| i\rangle\left(\epsilon_{k}+\epsilon_{i}\right)-\left\langle k\left|1 / r^{2}\right| i\right\rangle+\frac{1}{2}\left\langle k\left|1 / r^{3}\right| i\right\rangle\left(j(j+1)-l(l+1)-\frac{3}{4}\right)+\pi\langle k|\delta(\vec{r})| i\rangle\right],
$$

where $l$ and $j$ are the orbital and the total angular momentum quantum numbers. $\epsilon_{i}$ are Hydrogen energies.

Notice that $|i\rangle$ states can be labelled by $n, l, s=1 / 2$ (spin), $j$ and $m_{j}$ (total angular momentum projection on z) quantum numbers. We introduce now the states $|\mathbf{i}\rangle$ (boldfaced i), which are also eigenstates of $\hat{H}_{0}(Z=1)$, $\hat{L}^{2}$ and $\hat{S}^{2}$, but in addition they are eigenstates of $\hat{L}_{z}$ and $\hat{S}_{z}$ (z-components of $\hat{\vec{L}}$ and $\hat{\vec{S}}$ ) and generally not of $\hat{J}^{2}$ nor of $\hat{J}_{z}$. For the latter $|\mathbf{i}\rangle$ we need $n, l, m$ (orbital angular momentum projection on $z$ ), $s=1 / 2$, and $s_{z}$ (spin angular momentum projection on $z$ ) quantum numbers. We can expand $|i\rangle$ in terms of $|\mathbf{i}\rangle$ by means of the Clebsch-Gordan coefficients:

$$
|i\rangle=\sum_{\mathbf{i}}|\mathbf{i}\rangle\langle\mathbf{i} \mid i\rangle
$$

$\langle\mathbf{i} \mid i\rangle \equiv\left\langle l\right.$ sms $\left._{z} \mid j m_{j}\right\rangle$ are non-vanishing only for $|l-s| \leq$ $j \leq l+s$, and $m_{j}=m+s_{z}$.

The matrix elements entering Eq. (A2) after the expansion (A2a) are explicitly written as

$$
\begin{aligned}
\left\langle\mathbf{i}|\delta(\vec{r})| \mathbf{i}^{\prime}\right\rangle & =\delta_{l 0} \delta_{l l^{\prime}} \delta_{m m^{\prime}} \delta_{s_{z} s_{z}^{\prime}} \frac{1}{\pi} \frac{1}{\left(n n^{\prime}\right)^{3 / 2}}, \\
\left\langle\mathbf{i}\left|1 / r^{q}\right| \mathbf{i}^{\prime}\right\rangle & =\delta_{l l^{\prime}} \delta_{m m^{\prime}} \delta_{s_{z} s_{z}^{\prime}} \theta\left(n^{\prime}-1-l\right) 2^{2 l+2} \frac{\left(n n^{\prime}\right)^{l-q+1}}{\left(n+n^{\prime}\right)^{2 l+2-q+1}} \sqrt{(n-l-1) !\left(n^{\prime}-l-1\right) !(n+l) !\left(n^{\prime}+l\right) !} \\
& \times \sum_{k=0}^{n-l-1} \sum_{k^{\prime}=0}^{n^{\prime}-l-1} \frac{(-2)^{k+k^{\prime}} \frac{n^{k^{\prime} n^{\prime}}}{\left(n+n^{\prime}\right)^{k+k^{\prime}}} \Gamma\left(k+k^{\prime}+2 l+2-q+1\right)}{k ! k^{\prime} !(n-l-k-1) !\left(n^{\prime}-l-k^{\prime}-1\right) !(2 l+k+1) !\left(2 l+k^{\prime}+1\right) !} .
\end{aligned}
$$


1 R. Carcasses and A. Gonzalez, Phys. Rev. A 80, 024502 (2009).

2 A. Odriazola, A. Gonzalez and E. Räsänen, Phys. Rev. A 90, 052510 (2014).

3 D.A. Kirzhnits, Yu.E. Lozovik, and G.V. Shpatakovskaya, Sov. Phys. Usp. 18, 649 (1975); E.H. Lieb, Rev. Mod. Phys. 53, 603 (1981); L. Spruch, Rev. Mod. Phys. 63, 151 (1991).

4 G. Gil and A. Gonzalez, The NIST compilation of ionization potentials revisited (I): From He-like to Xe-like ions, arXiv:1405.6831 [physics.atom-ph].

${ }^{5}$ G. Gil and A. Gonzalez, The NIST compilation of ionization potentials revisited (II): From Ba-like to Th-like ions, in preparation.

6 A. Kramida, Yu. Ralchenko, J. Reader, and NIST ASD Team (2012). NIST Atomic Spectra Database (ver. 5.0), [Online]. Available: http://physics.nist.gov/asd [2013, March 4]. National Institute of Standards and Technology, Gaithersburg, MD.

7 A. Matulis and F.M. Peeters, J. Phys.: Cond. Matter 6, 7751 (1994); A. Gonzalez and I. Mikhailov, Int. J. Mod. Phys. B 11, 3469 (1997).

${ }^{8}$ G. Senatore and N.H. March, J. Chem. Phys. 83, 1232 (1985).

9 N.A. Cordero, N.H. March, J.A. Alonso, Phys. Rev. A 75, 012505 (2007).

10 G. Gil and A. Gonzalez, Mod. Phys. Lett. B 27, 1350178 (2013).

11 H. Hogreve, J. Phys. B 31, L439 (1998).

12 A.V. Sergeev and S. Kais, Int. J. Quant. Chem. 75, 533 (1999); S. Kais and P. Serra, Int. Rev. Phys. Chem. 19, 97 (2000).

13 D.K. Gridnev, J. Math. Phys. 53, 102108 (2012).

${ }^{14}$ H.A. Bethe, Phys. Rev. 76, 38 (1949); L.D. Landau and Ya.A. Smorodinsky, Lectures on Nuclear Theory (Plenum Press, New York, 1959).

15 Royal Society of Chemistry website http://www.rsc.org/periodic-table/. Data References: W.M. Haynes (ed.) CRC Handbook of Chemistry and Physics, CRC Press, Boca Raton, 2011; G.W.C. Kaye and T.H. Laby, Tables of Physical and Chemical Constants, Longman, Essex, 1995.

16 J. Katriel, J.P. Marques, P. Indelicato, A.M. Costa, M. C. Martins, J. P. Santos, F. Parente, Phys. Rev. A 90, 052519(2014); J. Katriel, G. Gaigalas, M. Puchalski, J.
Chem. Phys. 138, 224305 (2013); J. Katriel, M. Puchalski, K. Pachucki, Phys. Rev. A 86, 042508 (2012).

17 V.S. Popov, Sov. Phys. JETP 32, 3 (1971); W. Greiner, Relativistic quantum mechanics (Springer-Verlag, Berlin, 2000).

18 G. Gil, Stability of atoms in the anionic domain $(Z<$ $N$ ), Degree thesis (Faculty of Physics, University of Havana, 2012). Also in G. Gil, A. Gonzalez, arXiv:1309.3468 [physics.atom-ph].

19 As noticed in Ref. [9], the nonrelativistic behavior of the ionization potential with $Z$ is remarkably well described by considering only the first-order contribution to the $1 / Z$ expansion for not so large $Z$, e.g., $Z \approx 20$ in the case of Li-like and Be-like isoelectronic sequences.

20 Hund's rules are confirmed in the large- $Z$ limit by the calculations reported in the NIST database. ${ }^{6}$ In particular, we have checked the ground state electronic configuration and term symbol of the isoelectronic ions of $\mathrm{F}\left(1 \mathrm{~s}^{2} 2 \mathrm{~s}^{2} 2 \mathrm{p}^{5}\right.$ and ${ }^{2} P_{3 / 2}$, for $\left.Z \geq 9\right)$, Ne $\left(1 \mathrm{~s}^{2} 2 \mathrm{~s}^{2} 2 \mathrm{p}^{6}\right.$ and ${ }^{1} S_{0}$, for $\left.Z \geq 10\right)$, Co

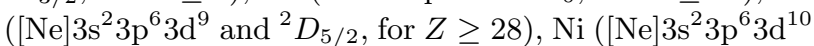
and ${ }^{1} S_{0}$, for $\left.Z \geq 29\right), \mathrm{Cu}\left([\mathrm{Ni}] 4 \mathrm{~s}\right.$ and ${ }^{2} S_{1 / 2}$, for $Z \geq 29$ ), $\operatorname{Pr}\left([\mathrm{Ni}] 4 \mathrm{~s}^{2} 4 \mathrm{p}^{6} 4 \mathrm{~d}^{10} 4 \mathrm{f}^{13}\right.$ and ${ }^{2} F_{7 / 2}$, for $\left.\left.Z \geq 80\right)\right)$ and $\mathrm{Nd}$ $\left([\mathrm{Ni}] 4 \mathrm{~s}^{2} 4 \mathrm{p}^{6} 4 \mathrm{~d}^{10} 4 \mathrm{f}^{14}\right.$ and ${ }^{1} S_{0}$, for $\left.Z \geq 84\right)$.

21 D. Layzer, Ann. Phys. (N.Y.) 8, 271 (1959).

22 B.H. Bransden and C.J. Joachain, Physics of atoms and molecules (Longman, Essex, 1983).

23 C. Froese, T. Brage and P. Jönsson, Computational Atomic Structure: An MCHF Approach (Institute of Physics Publishing, London, 1997).

24 The two-particle relativistic operator in the Breit-Pauli Hamiltonian behaves dimensionally as $\hat{W}_{\text {rel }}\left(\vec{r}_{1}, \vec{r}_{2}\right) \sim$ $Z \alpha^{2} /\left|\vec{r}_{1}-\vec{r}_{2}\right|^{3}$. Due to the scaling dictated by ThomasFermi, i.e., $\int d^{3} r \rho(\vec{r}) \sim N$ and $r \sim N^{2 / 3} / Z$, with $\rho(\vec{r})=\int d^{3} r_{1} d^{3} r_{2} \ldots d^{3} r_{N-1}\left|\left\langle\Psi^{(0)} \mid \vec{r}_{1} \vec{r}_{2} \ldots \vec{r}_{N-1} \vec{r}\right\rangle\right|^{2}$, the latter leads to $\hat{W}_{r e l} \sim \int d^{3} r_{1} \int d^{3} r_{2} \rho\left(\vec{r}_{1}\right) \rho\left(\vec{r}_{2}\right) / r_{12}^{3} \sim$ $N^{0} Z^{4} \alpha^{2}$. Because of the way ionization potential scale with $N$ for large $N$, i.e., $I_{p} \sim-d E_{g s} / d N$, the corrections to it coming from the $\hat{W}_{\text {rel }}$ would be zero. A more complete analysis will be published elsewhere. ${ }^{25}$

25 G. Gil, A. Gonzalez, A note on the large- $Z$ expansion for the ionization potential of atomic ions, in preparation. 